\title{
Adduct-based p-doping of Organic Semiconductors
}

Nobuya Sakai ${ }^{1}$, Ross Warren ${ }^{1}$, Fengyu Zhang ${ }^{2}$, Simantini Nayak ${ }^{3,4}$, Junliang Liu ${ }^{5}$, Sameer V. Kesava ${ }^{1}$, Yen-Hung Lin ${ }^{1}$, Himansu S. Biswal ${ }^{6}$, Xin Lin ${ }^{2}$, Chris Grovenor $^{5}$, Tadas Malinauskas ${ }^{7}$, Aniruddha Basu ${ }^{8}$, Thomas D. Anthopoulos ${ }^{8}$, Vytautas Getautis ${ }^{7}$ Antoine Kahn ${ }^{2}$, Moritz Riede ${ }^{1}$, Pabitra K. Nayak*1,9 and Henry J. Snaith*1

${ }^{1}$ Clarendon Laboratory, Department of Physics, University of Oxford, Parks Road, Oxford OX1 3PU, UK

${ }^{2}$ Department of Electrical Engineering, Princeton University, Princeton, New Jersey 08544, United States

${ }^{3}$ Department of Chemistry, University of Oxford, Inorganic Chemistry Laboratory, South Parks Road, Oxford OX1 3QR, UK

${ }^{4}$ Materials Chemistry Department, CSIR-Institute of Mineral and Materials Technology, Bhubaneswar, 751013, India

${ }^{5}$ Department of Materials, University of Oxford, Parks Road, Oxford OX1 3PH, UK

${ }^{6}$ School of Chemical Sciences, National Institute of Science Education and Research, 752050 Bhubaneswar, India

${ }^{7}$ Department of Organic Chemistry, Kaunas University of Technology, Radvilenu pl. 19, Kaunas LT-50254, Lithuania

8 King Abdullah University of Science and Technology (KAUST), KAUST Solar Center (KSC), Physical Sciences and Engineering Division (PSE), Material Science and Engineering Program (MSE), 23955-6900, Thuwal, Kingdom of Saudi Arabia

${ }^{9}$ TIFR Centre for Interdisciplinary Sciences, Tata Institute of Fundamental Research, Hyderabad, 500046, India

Email: pabitra.nayak@tifrh.res.in; henry.snaith@physics.ox.ac.uk

\section{Abstract}

Electronic doping of organic semiconductors is essential for their usage in highly efficient optoelectronic devices. While molecular and metal complex-based dopants have already enabled significant progress of devices based on organic semiconductors, there remains a need for clean, efficient and low-cost dopants if a widespread transition towards larger area organic electronic devices is to occur. Here, we report dimethyl sulfoxide adducts as $p$ dopants fulfilling these conditions for a range of organic semiconductors. These adduct-based dopants are compatible with both solution and vapour phase processing. We explore the 
doping mechanism and use the knowledge we gain to "decouple" the dopants from the choice of counterion. We demonstrate that asymmetric p-doping is possible using solution processing routes, and demonstrate its use in metal halide perovskite solar cells, organic thinfilm transistors and organic light-emitting diodes, showcasing the versatility of this doping approach.

\section{Main Text:}

Organic semiconductors are used in a wide range of cutting-edge technologies such as light emitting diodes (LEDs), field-effect transistors (FET) and photovoltaic (PV) devices due to their structural and functional tuneability ${ }^{1-5}$. Organic semiconductors usually have low charge carrier mobilities and low intrinsic conductivity, as compared to their inorganic counterparts. This, therefore, often necessitates doping to increase the number of charge carriers, leading to both filling traps and improvement in charge conductivity for device applications $^{6-9}$. A wide range of molecular dopants have been used for $p$-type and $n$-type doping of organic semiconductors ${ }^{10-14}$. However, challenges such as poor doping efficiency ${ }^{15,16}$ and doping instability ${ }^{17}$ still exist. Complex chemical structures of dopants require expensive synthesis and purification steps. Dopant molecules become the counterions during the doping process and remain in the host organic semiconductor ${ }^{14}$. Because the dopants and counterions are coupled, it is difficult to optimize their role separately. A further challenge is to achieve graded (asymmetric) doping in a device stack, or selective doping on one side of a charge conducting layer, especially using solution processing route $^{18}$, which is needed for better performance of devices. There is clearly the need for reliable and inexpensive dopants that circumvents the issues we have mentioned above associated with the present class of dopants.

Solutions of dimethyl sulfoxide (DMSO) with hydrobromic acid ( $\mathrm{HBr}$ ) have been used in the past for the oxidation of organic molecules during material synthesis, such as diphenylethane to benzyl, and acetophones to arylglyoxals ${ }^{19-21}$. They have also been used to alter the oxidization state of $\mathrm{Pd}^{2+}$ to $\mathrm{Pd}^{4+}$ in the synthesis of the inorganic compound $\mathrm{Cs}_{2} \mathrm{PdBr}_{6}{ }^{22}$ Here, we report that DMSO-HBr adducts can act as effective and clean $\mathrm{p}$-dopants for a wide range of organic semiconductors. Our approach is an in-situ formation of the dopant from commercially available low-cost chemicals, without any complex synthetic routes. The doping process is clean where the by-products and unreacted doping agents leave the thin-film. We further show that it is possible to select the counterion independently, unlike previously reported p-dopants. We also present a solution processible route to achieve asymmetric 
doping in organic semiconductor films, and demonstrate applications of the adduct-based doping process in metal halide perovskite-based solar cells, Organic thinfilm transistors (OTFTs) and OLEDs.

We start by testing the ability of our proposed agents to dope a variety of organic semiconductors, which are used as hole transporting material (HTM), in solution. In Fig. 1a and Supplementary Table 1, we show the chemical structure and ionization potentials of the selected HTMs, respectively. Here, we introduce to the HTM solution, a mixture of DMSO and $\mathrm{HBr}$ in 1:2 molar ratio, which we denote as the adduct forming agent (See Methods). Fig. 1b shows the absorbance spectra and the photographs of the HTM solutions before and after the addition of the adduct forming agent. We observe absorption features corresponding to oxidized organic molecules after the addition of the adduct forming agent into all the HTM solutions. We chose 2,2',7,7'-Tetrakis[N,N-di(4-methoxyphenyl)amino]-9,9'-spirobifluorene (Spiro-OMeTAD) as the testbed. After the addition of the adduct forming agent, we observe an absorption peak related to mono-cation radical Spiro-OMeTAD ${ }^{+}$at $\sim 520 \mathrm{~nm}$ (Fig. $1 \mathrm{~b}$ and Supplementary Fig. 1a) ${ }^{23,24}$. We estimate the doping efficacy, defined by the molar ratio of oxidized Spiro-OMeTAD to added DMSO, to be $17.5 \%$ (Supplementary Fig. 1b). To explore the possibility of doping the HTM using vapour phase techniques, we expose Spiro-OMeTAD thin-films to vapours of DMSO and $\mathrm{HBr}$ (Supplementary Fig. 2). As we show in Supplementary Fig. 3, there is a continuous increase in the absorbance corresponding to the oxidized species in the Spiro-OMeTAD thin-film with increasing vapour exposure time. This indicates that our doping strategy is compatible with both solution and vapour phase processing.

We show the current-voltage (I-V) curves of thin-film electrode-gap in-plane devices with and without doping (Supplementary Fig. 4) and we estimate the conductivity of the HTM films. We find a 2-3 order magnitude increase in the conductivity for all the HTMs with ionization energy (IE) $\leq 5.2 \mathrm{eV}$ after doping (Supplementary Table 1). In Supplementary Fig. 5 we show conductivity measurements for the material with the deepest highest occupied molecular orbital (HOMO) we found that we could dope, with an IE of $\sim 5.5 \mathrm{eV}$. We also evaluate the conductivity of the doped spiro-OMeTAD films using a 4-probe method ${ }^{25}$, which reach a maximum conductivity of $\sim 2 \times 10^{-3} \mathrm{~S} \mathrm{~cm}^{-1}$ (Fig 1 (c)) for both solution and vapor phase doping process. This is higher than what has been achieved for this HTM with other dopants ${ }^{26-}$ ${ }^{29}$ (Supplementary Fig. 6). To gain further insight into the doping effect, we perform ultraviolet photoelectron spectroscopy (UPS), X-ray photoelectron spectroscopy (XPS) and Kelvin probe based (KP) contact potential difference measurements on the HTM thin-films. We show the 
UPS spectra of the undoped and doped films of Spiro-OMeTAD in Fig. 1e and work function values measured by KP in Supplementary Fig. 7, respectively. As expected for p-type doping, we see an increase in the work function of the doped film and a shift of the Fermi levels closer to the HOMO level of the HTM. In Supplementary Fig. 8, we show the XPS spectra of the doped films. We observe $\mathrm{C}-1 \mathrm{~s}, \mathrm{O}-1 \mathrm{~s}$ and $\mathrm{N}-1 \mathrm{~s}$ peaks shifting to lower binding energies with an increase in the concentration of the adduct forming agent, which provides further confirmation of p-doping ${ }^{30}$. In Supplementary Fig. 9 we report similar UPS and XPS shifts upon doping for another HTM, $N, N, N^{\prime}$, $N^{\prime}$-Tetrakis(4-methoxyphenyl)benzidine (MeO-TPD).

The fact that neither DMSO nor HBr can dope the HTMs alone indicates that it is essential for both compounds to come together for the doping process to happen (Supplementary Fig. 10). Here, we investigate the doping mechanism by in-situ attenuated total reflection Fourier transform infrared spectroscopy (ATR-FTIR) and gas chromatographic mass spectrometry (GC-MS). In the FTIR spectrum of a DMSO solution in chlorobenzene, (Fig. 2a) we observe that the peak around $\sim 1060 \mathrm{~cm}^{-1}$, which corresponds to the absorbance of the $S=O$ bond ${ }^{31}$ of DMSO, broadens and shifts to lower wavenumber (around 1020-1050 $\mathrm{cm}^{-}$ $\left.{ }^{1}\right)$ after the addition of $\mathrm{HBr}$, indicating the lowering of the $\mathrm{S}=\mathrm{O}$ bond strength ${ }^{22,31}$. Based on quantum chemical calculations, we assign the peaks around $\sim 1035-1055 \mathrm{~cm}^{-1}$ to the formation of a molecular adduct between DMSO and $\mathrm{HBr}$ (Supplementary Fig. 11). We then add the Spiro-OMeTAD solution to the adduct solution and record the change in the absorbance spectra over time. We observe a continuous decrease in the peak intensity at $\sim 1050 \mathrm{~cm}^{-1}$, indicating the consumption of the DMSO-HBr adduct and breaking of the sulfuroxygen bond (Supplementary Fig. 12). Concurrently, we also record an increase in the absorbance of $\mathrm{OH}$ stretching around $~ 3400 \mathrm{~cm}^{-1}$ (Fig. 2b) ${ }^{32}$, indicating that $\mathrm{H}_{2} \mathrm{O}$ is one of the by-products of the process. Via GC-MS of the released gases during the doping process, we identify dimethyl sulfide (DMS) as another by-product (Supplementary Fig. 13). Based on this information, we propose the following mechanism for the doping: As the DMSO-HBr adduct accepts electron(s) from the HOMO level of the organic molecule, the activated DMSO reduces to DMS, one of the by-products. The oxidation states of sulfur in DMSO and DMS are 0 and -2 , respectively. The positive charge on the host organic cation is counterbalanced by the bromide anion from $\mathrm{HBr}$. The oxygen from DMSO and $\mathrm{H}$ from the $\mathrm{HBr}$ molecule then form $\mathrm{H}_{2} \mathrm{O}$, as another by-product. We show the probable mechanism schematically in Fig. 2c. Apart from the $\mathrm{Br}^{-}$counterion, which must be retained in the film to retain charge neutrality, we highlight that the doping process does not leave behind the by-products or unreacted dopants in the organic semiconductor matrix, which we confirm by the FTIR and XPS measurements on doped Spiro-OMeTAD films (Supplementary Fig. 14 -15 and Supplementary Note 1). 
However, similar systems such as DMSO-HCl and DMSO-HI adducts are not suitable for $\mathrm{p}$-type doping owing to their low efficiency and instability, respectively (Supplementary Fig. 16, 17, 18, and Supplementary Note 2).

Although the DMSO-HBr doping is clean and efficient, a critical factor for the utility of a doping process is that it must be stable under the conditions experienced during the manufacturing of additional layers in the device stack, particularly at elevated temperatures. We use conductivity and ellipsometry-based optical measurements to investigate the thermal stability of the doped films. To assess if the counter ion diffuses between layers, we constructed devices with a double layer of doped and undoped HTMs, comprising a bottomcontact gold electrode on a Si substrate (Fig. 3a), a spin-coated doped layer of MeO-TPD, followed by a thermally evaporated layer of intrinsic MeO-TPD.

If de-doping or migration of the doped organic molecule/counter ion occurs due to the thermal stress, we expect the conductivity and ellipsometric profiles of the films to change. ${ }^{17}$ As we show in Fig. 3b and Supplementary Fig. 19a, we do not observe any change in the conductivity of the sample, not any change in the ellipsometric profile (phase difference $\Delta$ and amplitude ratio $\Psi$ ) over a period of 100 hours at $50{ }^{\circ} \mathrm{C}$. The simulated profiles (See Supplementary Fig. 19b and Supplementary Note 3) for the doped and non-doped mixed films, are different than the observed profiles, confirming that the migration of the doped material (or counterion) does not happen during the $50^{\circ} \mathrm{C} 100$ hour stress test.

To further study the impact of doping on the morphology of the organic films, which is highly relevant for device applications, we perform atomic force microscopy (AFM) measurement of MeO-TPD films with different doping concentrations and following different levels of thermal stressing. We observe no significant changes in the topography (Fig. $3 \mathrm{~d}$ and Supplementary Fig. 20), for the doped films as compared to the undoped films, both before and after thermal stressing.

Despite being stable at $50{ }^{\circ} \mathrm{C}$, as we show in Fig. $3 \mathrm{c}$, when we raise the temperature to $85{ }^{\circ} \mathrm{C}$ we observe a decrease in the conductivity in films doped with DMSO-HBr adduct, indicating de-doping, presumably owing to the diffusion of $\mathrm{Br}^{-}$and subsequent escape from the matrix as $\mathrm{Br}_{2}$. $\mathrm{HBr}$ plays a dual role in the doping process - as an activator and then as a provider of the anion. Our doping mechanism should allow us to introduce other anions, specifically chosen for improved thermal stability of the doped material or for other properties, which would therefore be decoupled from the dopant. To demonstrate that such "decoupling" of the dopant and counterion is feasible, we choose a combination of 10camphorsulfonic acid (CSA), DMSO and $\mathrm{HBr}$. We estimate via a Van der Waals surface 
calculation that the counter ion, camphorsulfonate, is $\sim 8.5$ times bulkier than bromide ${ }^{33}$. The larger size of camphorsulfonate can likely reduce the diffusivity and volatility issues associated with bromide. We show the doping ability of the DMSO-HBr-CSA system in Spiro-OMeTAD and MeO-TPD by absorbance, conductivity, UPS and XPS studies (Supplementary Fig. 21-24). No doping occur in Spiro-OMeTAD solution when we add DMSO-CSA only, unlike DMSO-HBr (Supplementary Fig. 25). However, we only need to add a small volume ( 9 mol\% with respect to $\mathrm{CSA}$ ) of aqueous $\mathrm{HBr}$ to "activate" the doping process. We determine that the doping efficiency of the DMSO-HBr-CSA system is $\sim 22 \%$ (with respect to the added CSA molecules) for Spiro-OMeTAD from the optical absorbance change (Supplementary Fig. 21). For the films doped with DMSO-HBr-CSA, we observe no significant change in the conductivity (Fig. 3b and 3c) and morphology (Fig. 3d and Supplementary Fig. 26) with prolonged thermal stressing, even at $100{ }^{\circ} \mathrm{C}$. We present here only one example for the counterion exchange, but in principle, many other counterions tailored to specific needs could also be used. (See Supplementary Fig. 27 and Supplementary Note 4 and 5 for the mechanism of doping by DMSO-HBr-CSA system and its further applications)

One of the primary means to minimize contact resistance and create diode-like behaviour in semiconductor devices is to employ asymmetric doping, with the highly doped region in contact with the electrode material ${ }^{18,34-37}$. A key breakthrough for OLEDs has been the controlled $p$ and $n$-type doping of organic charge transport materials, and combining this with the deposition of multiple layers of different organic semiconductors upon one another ${ }^{38}$. For OLEDs, this is usually done via thermal evaporation of the organic semiconductors in vacuum. ${ }^{39,40}$

For solution-based processes, it is challenging to coat two subsequent layers on top of each other due to the common solubility of the host and dopants. Here, we explore the possibility of asymmetric doping (that is the doping is predominantly at one interface) via our adduct methods. To that end, we prepare "hole-only" device with a stack of FTO/Poly-TPD $(350 \mathrm{~nm}) / \mathrm{Au}$ where we introduce a CSA-DMSO-HBr layer (via solution process) either at the interface between Poly-TPD and Au or at the interface between Poly-TPD and FTO.

In Fig. 4a-c, we show the J-V curves of the hole-only devices where the interface and bulk are doped differently for each device. In Fig. 4a, the current density in the positive bias (that is, hole injection from the Au electrode) clearly is higher for the device with doped Poly-TPD:Au interface when compared to the undoped device. For the doped device, the current density increases with the dopant concentration in the doped layer, which we control via the concentration of the CSA (Supplementary Fig. 28a). Under negative bias (that is, hole 
injection from the FTO side), the current densities are similar for both the asymmetrically doped and undoped devices. As would be expected for the case of interface doping, we observe the opposite trend in the J-V curves (Fig. 4 (b) and Supplementary Fig. 28b) for the devices where the doping is introduced at the FTO: Poly-TPD interface, where we observe increased current density for the interface-doped device under negative bias. As we show in Fig. 4c, for homogeneous doping, the increase in the current densities are similar for both negative and positive bias.

To explore the positional localization of the space charge regions of the hole-only devices with interface doping, we perform capacitance-voltage measurements (Supplementary Fig. 29) and use Mott-Schottky analysis to estimate the acceptor density $\left(N_{A}\right)$ as a function of the depletion width $(w)$ from the conductive electrode-organic semiconductor junction (See Supplementary Information and Supplementary Note 6$)^{41-43}$. When profiling the acceptor density in the proximity of the doped interfaces, we find that the $N_{A}$ values in the devices for the doped Poly-TPD:Au interface (Fig. 4d) and doped FTO:Poly-TPD interface (Fig. 4e) exhibit a gradient profile. The $\mathrm{N}_{\mathrm{A}}$ follows a gradual decrease when the depletion width progresses further away from these doped interfaces, followed by a steep increase when the calculated depletion width approaches the width of the complete diode. In Fig. $4 \mathrm{f}$, we show the $N_{A}$ profile of the homogeneously doped devices. Compared to the undoped device (Supplementary Fig. 30 ), we see a homogenously higher $N_{A}$ value for the homogeneously doped device.

To further investigate the asymmetric doping, we perform Secondary lon Mass Spectrometry (SIMS) measurements of the doped and undoped films. Here we use silicon as a substrate. Since the HTM (MeO-TPD) does not contain sulphur, mapping of sulphur signal can be used to determine the distribution of the counter ions $\left(\mathrm{CSA}^{-}\right)$in the doped film. In Supplementary Fig. 31-32 we show the depth profiles of sulphur in undoped as well as doped films. For the films which we intended to dope at the top surface, we observe a significant enhancement of the sulphur signal at this interface (Supplementary Fig. 31b, Supplementary Note 7), and for the films doped at the buried HTM:Si interface, we observe sulphur enrichment at this buried interface (Supplementary Fig. 32). This enhancement of sulphur at the intended interfaces further confirms asymmetric doping.

We now demonstrate the utility of the doping methods in three types of optoelectronic devices which benefit from doped charge transport layers: organic thin film transistor (OTFT), perovskite solar cells and OLEDs (see Fig. 5a-c for the device architectures). 
We fabricate indacenodithiophene-benzothiadiazole ( $\mathrm{C}_{16}$ IDT-BT) based organic thin-film transistors (OTFTs) in a top-gate bottom-contact (TG-BC) device architecture (Fig. $5 a)^{44}$. Fig. $5 d$ shows the transfer current-voltage characteristics measured from OTFTs fabricated using pristine and moderately- and heavily-doped $\mathrm{C}_{16}$ IDT-BT based on the solutionprocessed DMSO-HBr adduct doping approach (denoted as DMSO-HBr (M) and DMSO-HBr $(H)$, respectively). We observe higher on-current levels and positively shifted turn-on voltages $\left(V_{O N}\right)$ due to p-doping. In Supplementary Table 3 and in Supplementary Fig. 33, we summarise the performance parameters from the transfer characteristics and provide the detailed analysis on the areal trap density ( $\Delta \mathrm{N}_{\mathrm{tr}}$, as compared to the undoped device) to and the trap concentration per unit energy $\left(D_{t r}\right)^{45}$ (see Supplementary Note 8 ). We achieve a clear improvement in field-effect mobility (Supplementary Table 3 ) by the adduct-based doping. We further confirm that the DMSO-HBr-CSA adduct doping assist hole transport in OTFTs in Supplementary Fig. 34 and Supplementary Table 4.

For perovskite $\left(\mathrm{FA}_{0.83} \mathrm{Cs}_{0.17} \mathrm{~Pb}\left(\mathrm{I}_{0.85} \mathrm{Br}_{0.15}\right)_{3}, \mathrm{FA}=\right.$ formamidinium $)$ solar cells, we use Spiro-OMeTAD as the HTM with the presented doping methods here and compare to other doping methods commonly used such as doping with Li-TFSI-oxygen or Co(III)TFSI (Supplementary Fig. 35 and Supplementary Table 5). In Fig. 5e, we show the J-V curves and steady-state power output (SPO) of our best-performing solar cells, where the HTM is doped with the DMSO-adduct based dopant or Li-TFSI-oxygen. We find that the devices where the HTM is doped with DMSO-HBr or DMSO-HBr-CSA show significantly improved power conversion efficiency (PCE) and SPO as compared to the commonly used doping methods (Fig. 5e). We summarize the PV performance parameters in Table 1 . In the Supplementary Information, we show additional information to corroborate the PV properties with our new doping, such as cross-sectional scanning electron micrograph of devices, external quantum efficiency (EQE), and forward and revere scan directions of the JV curves (see Supplementary Fig. 36 - 38 and Supplementary Table 6). We also demonstrate the doping capability of DMSO$\mathrm{HBr}$-CSA on Poly[bis(4-phenyl)(2,4,6-trimethylphenyl)amine (PTAA) in n-i-p perovskite solar cells, without tBP and Li-TFSI (Supplementary Fig. 39).

The Fermi level in the HTM monotonically shifts closer to the HOMO level onset with increasing dopant concentration (Fig. 1e). In the complete solar cell, the Fermi level alignment between the HTM and the quasi-Fermi level for holes in the perovskite absorber layer, and between the HTM and the Fermi level of the metallic electrode, are likely to improve with increased doping. Therefore, the increased $V_{\text {oc }}$ of the solar cells suggests an improved energetic alignment across these interfaces due to doping. The improvement in fill 
factor, is consistent with reduced series resistance in the cell, resulting from increased conductivity of the HTM. Therefore, both these effects, Fermi level deepening, and increased conductivity, appear to be contributing to the improved performance in the solar cells. (See Supplementary Note 9 and Supplementary Fig. 40 for discussion on increased short circuit current due to doping)

We fabricate OLEDs with the prototypical Tris-(8-hydroxyquinoline)aluminum ( $\left.\mathrm{Alq}_{3}\right)$ as the emitting material, sandwiched between MeO-TPD as the hole transport layer and bathophenanthroline (BPhen) as the electron transport layer (see Fig 5c). We show the luminance-voltage curves in Fig. $5 f$ and the $J-V$ curves in the inset and electroluminescence spectrum in Supplementary Fig. 41. We observe an increase in current density and luminance for OLEDs where the MeO-TPD layer is doped with DMSO-HBr or DMSO-HBr-CSA, in comparison to the undoped device. We attribute the improvement in the luminance to the improved charge injection with the doped HTMs. We also evaluate the performance of the OLED doped with F4TCNQ as a typical p-dopant (Supplementary Fig. 42). We find that the OLEDs prepared with the widely used p-dopant F4TCNQ perform comparably to our DMSO adduct-based dopant.

In conclusion, we have demonstrated a DMSO-adduct based p-doping scheme, applicable to a variety of organic HTMs ranging from small molecules to polymers. From understanding the doping mechanism, we have decoupled the dopant from the counter ions, allowing the electronic and physical properties and thermal stability of the doped HTM to be tuned separately. We also demonstrate that asymmetric doping is possible, using adduct based doping method. We have shown the usage of the doped organic layers in organic thin film transistors, perovskite solar cells and OLEDs. The p-doping method which we have presented here is not restricted to the DMSO-HBr adduct. In principle, it can be extended to other adduct systems where different sulfoxide containing molecules in combination with different activators should be feasible, highlighting an unexplored avenue to pursue for controlled doping of organic semiconductors.

\section{References:}

1. Granström, M. et al. Laminated fabrication of polymeric photovoltaic diodes. Nature 395, 257 (1998).

2. Halls, J. J. M. et al. Efficient photodiodes from interpenetrating polymer networks. Nature 376, 498 (1995).

3. Sirringhaus, H. et al. Two-dimensional charge transport in self-organized, highmobility conjugated polymers. Nature 401, 685 (1999). 
4. Tang, C. W. \& Vanslyke, S. A. Organic electroluminescent diodes. Appl. Phys. Lett. 51, 913-915 (1987).

5. Yu, G., Gao, J., Hummelen, J. C., Wudl, F. \& Heeger, A. J. Polymer Photovoltaic Cells: Enhanced Efficiencies via a Network of Internal Donor-Acceptor Heterojunctions. Science. 270, 1789 - 1791 (1995).

6. Blochwitz, J., Pfeiffer, M., Fritz, T. \& Leo, K. Low voltage organic light emitting diodes featuring doped phthalocyanine as hole transport material. Appl. Phys. Lett. 73, 729731 (1998).

7. Maennig, B. et al. Controlled p -type doping of polycrystalline and amorphous organic layers : Self-consistent description of conductivity and field-effect mobility by a microscopic percolation model. 64, 1-9 (2001).

8. Godet, C. Variable range hopping revisited : the case of an exponential distribution of localized states. J. Non. Cryst. Solids 302, 333-338 (2002).

9. Rubel, O., Baranovskii, S. D., Thomas, P. \& Yamasaki, S. Concentration dependence of the hopping mobility in disor- dered organic solids. phys. stat. sol. 171, 168-171 (2004).

10. Blochwitz, J. et al. Interface electronic structure of organic semiconductors with controlled doping levels. Org. Electron. 2, 97-104 (2001).

11. Gao, W. \& Kahn, A. Controlled p-doping of zinc phthalocyanine by coevaporation with tetrafluorotetracyanoquinodimethane: A direct and inverse photoemission study. Appl. Phys. Lett. 79, 4040-4042 (2001).

12. Harada, K. et al. Organic Homojunction Diodes with a High Built-in Potential: Interpretation of the Current-Voltage Characteristics by a Generalized Einstein Relation. Phys. Rev. Lett. 94, 36601 (2005).

13. Chan, C. K., Zhao, W., Barlow, S., Marder, S. \& Kahn, A. Decamethylcobaltocene as an efficient n-dopant in organic electronic materials and devices. Org. Electron. 9, 575581 (2008).

14. Lin, X. et al. Beating the thermodynamic limit with photo-activation of $n$-doping in organic semiconductors. Nat. Mater. 16, 1209 (2017).

15. Tietze, M. L. et al. Elementary steps in electrical doping of organic semiconductors. Nat. Commun. 9, 1182 (2018).

16. Tietze, M. L., Burtone, L., Riede, M., Lüssem, B. \& Leo, K. Fermi level shift and doping efficiency in $p$-doped small molecule organic semiconductors : A photoelectron spectroscopy and theoretical study. Phys. Rev. B 035320, 1-12 (2012). 
17. Li, J. et al. Measurement of Small Molecular Dopant F4TCNQ and C60F36 Diffusion in Organic Bilayer Architectures. ACS Appl. Mater. Interfaces 7, 28420-28428 (2015).

18. Kolesov, V. A. et al. Solution-based electrical doping of semiconducting polymer films over a limited depth. Nat. Mater. 16, 474-481 (2017).

19. Yusubov, M. S., Filimonov, V. D. \& Ogorodnikov, V. D. Dimethyl sulfoxide-hydrobromic acid as a novel reagent for convenient oxidation on a preparative scale of stilbenes and some derivatives of diphenylethane to benzils. Bull. Acad. Sci. USSR, Div. Chem. Sci. 40, 766-770 (1991).

20. Lee, T. V. Oxidation Adjacent to Oxygen of Alcohols by Activated DMSO Methods. Compr. Org. Synth. 7, 291-303 (1991).

21. Floyd, M. B., Du, M. T., Fabio, P. F., Jacob, L. A. \& Johnson, B. D. The oxidation of acetophenones to arylglyoxals with aqueous hydrobromic acid in dimethyl sulfoxide. J. Org. Chem. 50, 5022-5027 (1985).

22. Sakai, N. et al. Solution-Processed Cesium Hexabromopalladate(IV), Cs2PdBr6, for Optoelectronic Applications. J. Am. Chem. Soc. 139, 6030-6033 (2017).

23. Cappel, U. B., Daeneke, T. \& Bach, U. Oxygen-Induced Doping of Spiro-MeOTAD in Solid-State Dye-Sensitized Solar Cells and Its Impact on Device Performance. Nano Lett. 12, 4925-4931 (2012).

24. Burschka, J. et al. Tris(2-(1H-pyrazol-1-yl)pyridine)cobalt(III) as p-Type Dopant for Organic Semiconductors and Its Application in Highly Efficient Solid-State DyeSensitized Solar Cells. J. Am. Chem. Soc. 133, 18042-18045 (2011).

25. Planells, M. et al. Diacetylene bridged triphenylamines as hole transport materials for solid state dye sensitized solar cells. J. Mater. Chem. A 1, 6949-6960 (2013).

26. Abate, A. et al. Protic lonic Liquids as p-Dopant for Organic Hole Transporting Materials and Their Application in High Efficiency Hybrid Solar Cells. J. Am. Chem. Soc. 135, 13538-13548 (2013).

27. Pellaroque, A. et al. Efficient and Stable Perovskite Solar Cells Using Molybdenum Tris(dithiolene)s as p-Dopants for Spiro-OMeTAD. ACS Energy Lett. 2, 2044-2050 (2017).

28. Nguyen, W. H., Bailie, C. D., Unger, E. L. \& McGehee, M. D. Enhancing the HoleConductivity of Spiro-OMeTAD without Oxygen or Lithium Salts by Using Spiro(TFSI)2 in Perovskite and Dye-Sensitized Solar Cells. J. Am. Chem. Soc. 136, 10996-11001 (2014).

29. Chen, C. et al. Cu(II) Complexes as p-Type Dopants in Efficient Perovskite Solar Cells. ACS Energy Lett. 2, 497-503 (2017). 
30. Ono, L. K. et al. Air-Exposure-Induced Gas-Molecule Incorporation into SpiroMeOTAD Films. J. Phys. Chem. Lett. 5, 1374-1379 (2014).

31. Fawcett, W. R. \& Kloss, A. A. Solvent-Induced Frequency Shifts in the Infrared Spectrum of Dimethyl Sulfoxide in Organic Solvents. J. Phys. Chem. 100, 2019-2024 (1996).

32. Wallace, V. M., Dhumal, N. R., Zehentbauer, F. M., Kim, H. J. \& Kiefer, J. Revisiting the Aqueous Solutions of Dimethyl Sulfoxide by Spectroscopy in the Mid- and NearInfrared: Experiments and Car-Parrinello Simulations. J. Phys. Chem. B 119, 1478014789 (2015).

33. Zhao, Y. H., Abraham, M. H. \& Zissimos, A. M. Fast Calculation of van der Waals Volume as a Sum of Atomic and Bond Contributions and Its Application to Drug Compounds. J. Org. Chem. 68, 7368-7373 (2003).

34. Kang, K. et al. 2D coherent charge transport in highly ordered conducting polymers doped by solid state diffusion. Nat. Mater. 15, 896 (2016).

35. Ávila, J. et al. High voltage vacuum-deposited $\mathrm{CH} 3 \mathrm{NH} 3 \mathrm{PbI3}-\mathrm{CH} 3 \mathrm{NH} 3 \mathrm{Pbl} 3$ tandem solar cells. Energy Environ. Sci. 11, 3292-3297 (2018).

36. Abdi-Jalebi, M. et al. Charge extraction via graded doping of hole transport layers gives highly luminescent and stable metal halide perovskite devices. Sci. Adv. 5, eaav2012 (2019).

37. Wanlass, M. Systems and methods for advanced ultra-high-performance InP solar cells. (2017).

38. Walzer, K., Maennig, B., Pfeiffer, M. \& Leo, K. Highly Efficient Organic Devices Based on Electrically Doped Transport Layers. Chem. Rev. 107, 1233-1271 (2007).

39. Pfeiffer, M., Beyer, A., Fritz, T. \& Leo, K. Controlled doping of phthalocyanine layers by cosublimation with acceptor molecules: A systematic Seebeck and conductivity study. Appl. Phys. Lett. 73, 3202-3204 (1998).

40. Nollau, A., Pfeiffer, M., Fritz, T. \& Leo, K. Controlled n-type doping of a molecular organic semiconductor: Naphthalenetetracarboxylic dianhydride (NTCDA) doped with bis(ethylenedithio)-tetrathiafulvalene (BEDT-TTF). J. Appl. Phys. 87, 4340-4343 (2000).

41. Kirchartz, T. et al. Sensitivity of the Mott - Schottky Analysis in Organic Solar Cells. J. Phys. Chem. C 116, 7672-7680 (2012).

42. Deledalle, F. et al. Understanding the Effect of Unintentional Doping on Transport Optimization and Analysis in Efficient Organic Bulk-Heterojunction Solar Cells. Phys. Rev. X 5, 11032 (2015). 
43. Zonno, I., Martinez-Otero, A., Hebig, J. \& Kirchartz, T. Understanding Mott-Schottky Measurements under Illumination in Organic Bulk Heterojunction Solar Cells. Phys. Rev. Appl. 7, 034018 (2017).

44. Lin, Y.-H. et al. Deciphering photocarrier dynamics for tuneable high-performance perovskite-organic semiconductor heterojunction phototransistors. Nat. Commun. 10, 4475 (2019).

45. Lin, Y.-H. et al. Hybrid organic-metal oxide multilayer channel transistors with high operational stability. Nat. Electron. 2, 587-595 (2019).

46. Anaraki, E. H. et al. Highly efficient and stable planar perovskite solar cells by solution-processed tin oxide. Energy Environ. Sci. 9, 3128-3134 (2016).

47. Huang, Q. et al. Highly efficient top emitting organic light-emitting diodes with organic outcoupling enhancement layers. Appl. Phys. Lett. 88, 113515 (2006).

48. Sakai, N. et al. Adduct-based p-doping of organic semiconductors, Oxford University Research Archive. (2021). doi:10.5287/bodleian:zrMDxRzzB

\section{Acknowledgements:}

This research has mainly received funding from the European Commission (PERTPVagreement number 763977) and EPSRC (EP/M005143/1) and EP/S004947/1

M.R. has received funding from the EC FP 7 MSCA - Career Integration Grant (630864) and EPSRC (EP/M015173/1). R.W. is supported by EPSRC CDT Plastic Electronics (EP/L016702/1). P.N. acknowledges the support from the Department of Atomic Energy, Government of India, under Project Identification No. RTI 4007 and SERB India core research grant (CRG/2020/003877). F.Z., X.L. and A.K. acknowledge funding from National Science Foundation under grants DMR-1506097 and DMR-1807797. S.N. acknowledges Marie Skłodowska-Curie actions individual fellowships (grant agreement number 659306) and start up grant from CSIR-IMMT, India. T.M. and V.G. acknowledge funding from European Regional Development Fund (project No 01.2.2-LMT-K-718-03-0040) under grant agreement with the Research Council of Lithuania (LMTLT). We thank I. McPherson for his help in mass spectrometry measurement

Contributions: NS and PN conceived and executed the initial proof of concept experiments and unravel the mechanism of doping. PN proposed dopant system. HJS proposed the asymmetric doping and NS designed and performed the experiments. NS and RW performed the conductivity measurements. NS, RW performed the doping stability test under the supervision of MR. SK performed the ellipsometry measurements, analyses and simulation under the supervision of MR. RW fabricated OLEDs under supervision of MR and NS fabricated 
all other the devices used in this work. SN and PN performed the ATR-FTIR measurements. FZ and $X L$ did the UPS, XPS and Kelvin probe measurements under the supervision of AK. FZ did the AFM measurements. JL did the Nano-SIMS measurements with inputs from PN and NS. C G planned and helped interpreting the Nano-SIMS measurements. NS and Y-HL performed the capacitance-voltage measurements. HB performed the quantum chemical calculations. TM conducted synthesis of the HTM V886, VG supervised the synthesis. AB fabricated OTFTs and performed electrical characterisation under the supervision of TDA. TDA, Y-HL and AB interpreted the results and provided the analysis of OTFTs.

NS and PN wrote the first draft. All the authors contributed to the analysis of the results and discussion of the content and revisions of the manuscript. PN and HJS supervised the project. Competing interest: A patent based on this work has been filed (International application number: PCT/GB2018/053014) by the University of Oxford. H.J.S. is a co-founder of Oxford PV Ltd and Helio Display Materials. The remaining authors declare no competing interests. 


\section{Figure Legends:}

Figure 1| Doping ability of DMSO-HBr adduct for various hole-transporting materials. a, Chemical structures of hole-transporting materials (N2,N2,N2',N2',N7,N7,N7',N7'-octakis(4methoxyphenyl)-9,9'-spirobi[9H-fluorene]-2,2',7,7'-tetramine (Spiro-OMeTAD), N,N,N', $N^{\prime}$ tetrakis(4-methoxyphenyl)benzidine (MeO-TPD), 1,2-bis[3,6-(4,4dimethoxydiphenylamino)-9H-carbazol-9-methyl]benzene (V886), poly(3-hexylthiophene2,5-diyl) (P3HT), poly[N,N'-bis(4-butylphenyl)-N, $\mathrm{N}^{\prime}$-bisphenylbenzidine] (Poly-TPD)) and poly[bis(4-phenyl)(2,4,6-trimethylphenyl)amine] (PTAA). b, UV-vis absorption spectra of organic HTMs in chlorobenzene $\left(1-9 \times 10^{-6} \mathrm{M}\right)$ before (dotted line) and after (solid) the addition of the adduct forming agent. Insets: photographs of vials containing the neat HTM solution (left) and HTM solution with the adduct forming agent (right). c, d, Conductivity of the SpiroOMeTAD thin-film doped with DMSO-HBr by either adding the dopant directly to the HTM solution (c: solution process) or exposing the thin films to DMSO-HBr vapor (d: vapor process). e, UPS spectra of the un-doped and doped (solution process) Spiro-OMeTAD thin-films.

Figure 2| Mechanism of doping by DMSO-HBr adduct. $\mathbf{a}, \mathbf{b}, \mathrm{FTIR}$ spectrum of DMSO-HBr mixture in chlorobenzene, and after the addition of Spiro-OMeTAD showing the consumption of the adduct and formation of $\mathrm{H}_{2} \mathrm{O}$ during the doping process. (a) the region of $\mathrm{S}=\mathrm{O}$ absorbance (b) the region of the $\mathrm{OH}$ stretching absorbance. c, Proposed mechanism of the doping process.

Figure 3| Thermal stability of the doped MeO-TPD films. a, Chemical structure of camphorsulfonic acid (CSA) and schematic illustration of geometry to evaluate thermal stability of dopants by the conductivity measurement. $\mathbf{b}$, Conductivity of the MeO-TPD films doped with DMSO-HBr and DMSO-HBr-CSA after thermal stressing at $50{ }^{\circ} \mathrm{C}$ for 100 hours. C, Conductivity (at room temperature) of undoped MeO-TPD films and doped MeO-TPD film with DMSO-HBr and DMSO-HBr-CSA after stressing at different temperatures for 10 minutes on a hotplate in a nitrogen filled glove box. $\mathbf{d}$, Atomic force microscope (AFM) topography images of the undoped and doped MeO-TPD films. The AFM images are taken after the films are incubated at 20,50 and $85^{\circ} \mathrm{C}$ for 10 minutes. The scale bar is $500 \mathrm{~nm}$. Root Mean Square (RMS) height variation is evaluated over $2 \times 2 \mu \mathrm{m}^{2}$ in each AFM image.

Figure 4| Asymmetric doping in hole-only devices. a, b, c, Current density-voltage curves of the hole-only devices (a) doped at Poly-TPD: Au interface (b) at FTO: Poly-TPD interface, and (c) homogeneous doping. d, e, $\mathbf{f}$ Acceptor density $\left(N_{A}\right)$ as a depletion width $(w)$ of hole-only 
devices (d) doped at the Poly-TPD:Au interface, (e) doped at the FTO:Poly-TPD interface, (f) homogeneous doping.

Figure 5| Usage of adduct-based dopants in optoelectronic devices. a-c, Schematic of the organic thin film transistor (OTFT: a), perovskite photovoltaic device (b) and the organic light emitting device (OLED: c).d, Current-voltage characteristics of DMSO-HBr-doped C16 IDT-BT based OTFT e, Current density-voltage curves of the solar cells with different dopants and counterions, Inset: stabilized power output (SPO) for the respective perovskite solar cells at a fixed maximum power point voltage. f, luminescence-voltage curve for the OLEDs with different dopants and counterions, inset: Current density-voltage curves of the OLEDs.

Table 1| Device performance parameters of perovskite solar cells doped with DMSOadducts based dopant and Li-TFSI-Oxygen.

\begin{tabular}{lllllll}
\hline Doping method & $\begin{array}{l}\mathrm{J}_{\mathrm{sc}} \\
\left(\mathrm{mA} \mathrm{cm}{ }^{-2}\right)\end{array}$ & $\mathrm{V}_{\mathrm{oc}}(\mathrm{V})$ & $\mathrm{FF}$ & PCE (\%) & $\begin{array}{l}\text { Rs } \\
(\mathrm{Ohm} .)\end{array}$ & SPO (\%) \\
\hline Li-TFSI-oxygen & 22.8 & 1.08 & 0.76 & 18.7 & 39.9 & 18.2 \\
DMSO-HBr & 23.2 & 1.14 & 0.79 & 21.1 & 38.9 & 19.8 \\
DMSO-HBr-CSA & 23.5 & 1.11 & 0.81 & 21.3 & 32.5 & 20.1 \\
\hline
\end{tabular}




\section{Methods:}

\section{General experimental}

Preparation of DMSO-HBr adduct forming agent:

$150 \mu \mathrm{L}$ of DMSO (Aldrich; anhydrous, $\geq 99.9 \%$ ) was added into $500 \mu \mathrm{L}$ of aqueous $\mathrm{HBr}$ (Aldrich: $48 \mathrm{wt} . \%)$ and the solution was mixed by a vortex mixer for $1 \mathrm{~min}$. The molar ratio of DMSO and $\mathrm{HBr}$ is $\sim 1: 2$.

Doping of HTM in solution by DMSO-HBr adduct: 1-5 $\mu \mathrm{L}$ of adduct forming agent was added to the $1 \mathrm{~mL}$ HTM solutions (Small molecules $\sim 20 \mu \mathrm{M}$ and Polymers $\sim 25 \mu \mathrm{g} / \mathrm{ml}$ ) in chlorobenzene or toluene and the solution was mixed by a vortex mixer. The solution was kept at room temperature for 20 minutes. The concentration of the adduct forming agent in the HTM solutions was varied by adding different amounts of adduct forming agent.

Preparation of doped HTM thin films by DMSO-HBr adduct in a solution process: $10 \mu \mathrm{L}$ of adduct forming agent was added to $2.5 \mathrm{~mL}$ of 2,2(7,7(-tetrakis-(N,N-di-pmethoxyphenylamine)9,9(-spirobifluorene))) (0.28 mM Spiro-OMeTAD(: Luminescence technology)) solution in chlorobenzene (Aldrich; anhydrous, $\geq 99.9 \%$ ). The solution was mixed by a vortex mixer for $1 \mathrm{~min}$ and then kept for 20 mins at room temperature to prepare a heavily doped Spiro-OMeTAD solution. 3-15 $\mu \mathrm{L}$ of the heavily doped Spiro-OMeTAD solution was added to $1 \mathrm{~mL}$ of un-doped Spiro-OMeTAD solution (70 $\mathrm{mM}$ ) in chlorobenzene and the solution was kept for at least 15 minutes before thin film preparation. The resulting solution was spin coated onto the substrate at $2500 \mathrm{rpm}$ for $45 \mathrm{sec}$ (acceleration: $500 \mathrm{rpm}$ ) to prepare the thinfilm. A typical example for the preparation of doped thin films Spiro-OMeTAD is provided here. Similar protocols were adapted for other HTMs: The concentration of adduct forming agent in the HTM solution was varied by changing the amount of adduct forming agent in the HTM solution.

Preparation of doped HTM thin films by DMSO-HBr adduct in a vapor phase process: SpiroOMeTAD solution in chlorobenzene $(70 \mathrm{mM})$ was spin coated onto the substrate at $2500 \mathrm{rpm}$ for $45 \mathrm{sec}$ (acceleration: $500 \mathrm{rpm}$ ). $1.5 \mu \mathrm{L}$ of DMSO and $5 \mu \mathrm{L}$ of aqueous $\mathrm{HBr}$ were put in separate crucibles in a low vacuum chamber (PELCO 2245; pressure $\sim 70 \mathrm{kPa}$ ) along with the un-doped Spiro-OMeTAD thin films on glass substrates. The petri-dish containing DMSO was heated at $55^{\circ} \mathrm{C}$ in the vacuum chamber while the substrate and $\mathrm{HBr}$ container remained at the ambient temperature. The exposure time was varied to control the doping concentration 
in the film. We show the schematic illustration of the set up for the vapor process doping in Supplementary Figure 2.

Preparation of doped MeO-TPD thinfilms by DMSO-HBr adduct in a solution process: $150 \mu \mathrm{L}$ of DMSO was added into $500 \mu \mathrm{L}$ of aqueous $\mathrm{HBr}$ (48 wt. \%) and the solution was mixed by a vortex mixer for $1 \mathrm{~min}$. $10 \mu \mathrm{L}$ of the DMSO-HBr solution (adduct forming agent) was added to $2.5 \mathrm{~mL}$ of MeO-TPD (0.32 mM) solution in chlorobenzene. The solution was mixed by a vortex mixer for $1 \mathrm{~min}$ and then kept for $20 \mathrm{~min}$ at room temperature to prepare a heavily doped MeO-TPD solution. $50 \mu \mathrm{L}$ of the heavily doped MeO-TPD solution was added to $150 \mu \mathrm{L}$ of undoped MeO-TPD solution $(80 \mathrm{mM})$ in chlorobenzene and the solution was kept for at least 10 minutes before the thin film preparation. The doped MeO-TPD solution was spin-coated onto the substrate at $2500 \mathrm{rpm}$ for $45 \mathrm{sec}$ (acceleration: $500 \mathrm{rpm}$ ).

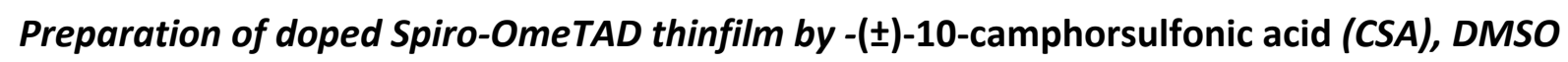
and $\mathrm{HBr}$ :

$30 \mathrm{mg}$ of CSA (Aldrich, ( \pm )-10-camphorsulfonic acid, 98\%) was dissolved in $10 \mu \mathrm{L}$ of DMSO and $300 \mu \mathrm{L}$ of chlorobenzene. The solution was vortexed until CSA was dissolved completely. $3 \mu \mathrm{L}$ of aqueous $\mathrm{HBr}$ (48 wt\%) was added to the CSA solution and the solution was vortexed for 30 seconds. $50 \mu \mathrm{L}$ of DMSO-HBr-CSA solution was added into $500 \mu \mathrm{L}$ of Spiro-OMeTAD solution (7.78 $\mathrm{mM}$ ) in chlorobenzene to form a heavily doped Spiro-OMeTAD solution. $5-50 \mu \mathrm{L}$ of heavily doped Spiro-OMeTAD solution was added to the un-doped Spiro-OMeTAD chlorobenzene solution (70 mM) and was kept for $15 \mathrm{~min}$ before the preparation of thin films. The Spiro-OMeTAD solution was spin-coated onto the perovskite layer at $2500 \mathrm{rpm}$ for $45 \mathrm{sec}$ (acceleration: $500 \mathrm{rpm}$ ). The molar ratio of DMSO, $\mathrm{HBr}$ and CSA was 1:0.09:1.

Preparation of doped MeO-TPD thin film by DMSO-HBr-CSA: $30 \mathrm{mg}$ of CSA was dissolved in $10 \mu \mathrm{L}$ of DMSO and $300 \mu \mathrm{L}$ of chlorobenzene. The solution was vortexed until CSA was dissolved completely. $3 \mu \mathrm{L}$ of aqueous $\mathrm{HBr}$ (48 wt\%) was added to the CSA solution and the solution was vortexed for a further 30 seconds. $50 \mu \mathrm{L}$ of DMSO-HBr-CSA solution was added into $500 \mu \mathrm{L}$ of MeO-TPD solution $(6.87 \mathrm{mM})$ in chlorobenzene to prepare a heavily doped MeO-TPD solution. $5 \mu \mathrm{L}$ of the heavily doped MeO-TPD solution was added to $105 \mu \mathrm{L}$ of undoped MeO-TPD chlorobenzene solution ( $80 \mathrm{mM}$ ) and was kept for $10 \mathrm{~min}$ before the preparation of thin films. The MeO-TPD solution was spin-coated on to the substrate at 2500 rpm for $45 \mathrm{sec}$ (acceleration: $500 \mathrm{rpm}$ ).

Asymmetric doping with DMSO-HBr-CSA: 
Preparation of substrate: FTO (F-doped tin oxide) -coated glass sheets (TEC7, 7 $\Omega /$ sq, Pilkington) were etched with zinc powder and $2 \mathrm{M} \mathrm{HCl}$ to obtain the required electrode pattern. The sheets were then washed with a $2 \%$ Hellmanex solution in water followed by sonication for 10 minutes and then washed with deionized water, acetone, ethanol and isopropanol. The last traces of organic residues were removed by oxygen plasma cleaning for 10 minutes.

Preparation of the stock solution of CSA-DMSO-HBr: $30 \mathrm{mg}$ of CSA was dissolved in a solution of $20 \mu \mathrm{L}$ of DMSO, $10 \mu \mathrm{L}$ of aqueous $\mathrm{HBr}$ (48 wt\%), $250 \mu \mathrm{L}$ of acetonitrile (Aldrich, anhydrous, 99.8\%) and $50 \mu \mathrm{L}$ 2-Ethoxyethanol (Aldrich, 99\%). The molar ratio of DMSO, HBr and CSA was always kept at $\sim 2:$ 0.3:1. The CSA-DMSO-HBr stock solution was diluted in acetonitrile and 2Ethoxyethanol mixture (5:1 volume ratio) to change the concentration of CSA.

Preparation of hole only device with the doping at HTM/ Au interface: Poly[N, $\mathrm{N}^{\prime}-\mathrm{bis}(4-$ butylphenyl)-N,N'-bisphenylbenzidine] (Poly-TPD) solution (50 mg/1mL toluene) was spincoated on to FTO substrate at $2000 \mathrm{rpm}$ for $45 \mathrm{sec}$ (acceleration: $500 \mathrm{rpm}$ ) and the film was annealed on a hot plate at $100{ }^{\circ} \mathrm{C}$ for 5 minutes. The CSA-DMSO-HBr stock solution (concentration of CSA is $\sim 0.25-25 \mathrm{mM}$ ) was spin-coated on the undoped Poly-TPD layer at $4000 \mathrm{rpm}$ for $45 \mathrm{sec}$ (acceleration: $500 \mathrm{rpm}$ ). The treated thin film was annealed on a hot plate at $100{ }^{\circ} \mathrm{C}$ for 5 minutes. Note that this annealing process is enough for the unreacted chemicals, by-products and unstable dopant counter ions to leave the organic semiconductor matrix. To control the concentration of dopant on the poly TPD surface, the stock solution of CSA-DMSO-HBr solution was diluted in acetonitrile and 2-Ethoxyethanol (5:1 volume ratio) before the spin coating on the undoped Poly-TPD layer. The top Au layer was prepared by thermal evaporation of $\mathrm{Au}$ in vacuum $\left(5 \times 10^{-6}\right.$ Torr).

Preparation of hole only device with the doping at FTO/HTM interface: The CSA-DMSO-HBr stock solution (concentration of CSA is $\sim 0.5-50 \mathrm{mM}$ ) was spin-coated on a precleaned FTO substrate. Poly-TPD solution (50 mg in $1 \mathrm{~mL}$ of toluene) was spin-coated on the CSA-DMSO$\mathrm{HBr}$ coated FTO substrate at $2000 \mathrm{rpm}$ for $45 \mathrm{sec}$ (acceleration: $500 \mathrm{rpm}$ ) and the film was annealed on the hot plate at $100{ }^{\circ} \mathrm{C}$ for 5 minutes. To control the concentration of dopant at the FTO: Poly TPD film interface, the CSA-DMSO-HBr stock solution was diluted in acetonitrile and 2-Ethoxyethanol (5:1 volume ratio), prior to the spin coating.

Preparation of hole only device with the uniform doping at HTM: 50 uL of the CSA-DMSO-HBr stock solution ( $40 \mathrm{mM}$ ) was added into $1 \mathrm{~mL}$ Poly-TPD solution $(50 \mathrm{mg} / \mathrm{mL})$. Poly-TPD was 
deposited on the FTO substrate at 2000 rpm for 45 seconds (acceleration: $500 \mathrm{rpm}$ ) and the film was annealed on the hot plate at $100{ }^{\circ} \mathrm{C}$ for $10 \mathrm{~min}$.

Fabrication of Organic transistors: $4 \mu \mathrm{L}$ of DMSO-HBr adduct forming agent was added to 1 $\mathrm{mL}$ of $\mathrm{C}_{16}$ IDT-BT solution $\left(0.05 \mathrm{mg} / \mathrm{ml}\right.$ in chlorobenzene). The $\mathrm{C}_{16}$ IDT-BT/DMSO-HBr solution was again mixed by a vortex mixer for $1 \mathrm{~min}$ and then kept for $20 \mathrm{~min}$ at room temperature as the doped $\mathrm{C}_{16}$ IDT-BT stock solution. $30 \mu \mathrm{L}$ (moderately-doped) and $50 \mu \mathrm{L}$ (heavily-doped) of this doped $\mathrm{C}_{16}$ IDT-BT stock solution was added to $150 \mu \mathrm{L}$ of pristine $\mathrm{C}_{16}$ IDT-BT solution $(13.33 \mathrm{mg} / \mathrm{ml}$ in chlorobenzene). Finally, in order to obtain a final solution concentration of $10 \mathrm{mg} / \mathrm{ml}$ for preparing $C_{16}$ IDT-BT OTFTs, the pristine $C_{16}$ IDT-BT solution $(0.05 \mathrm{mg} / \mathrm{ml}$ in chlorobenzene) was added into both the as-prepared moderately-doped and heavily-doped $\mathrm{C}_{16}$ IDT-BT solutions. The solutions were kept for $>10$ minutes before the thin film deposition.

OTFTs were fabricated in a Top Gate Bottom Contact (TG-BC) architecture on $2.54 \times 2.54 \mathrm{~cm}^{2}$ glass substrates. The source and drain electrodes of $\mathrm{Al} / \mathrm{Au}(5 / 35 \mathrm{~nm})$ and $\mathrm{Al}(40 \mathrm{~nm})$ for $\mathrm{p}$ type and n-type devices were thermally deposited through metal shadow masks on the glass substrates, respectively. The channel width and length employed were $1000 \mu \mathrm{m}$ and $30 \mu \mathrm{m}$, respectively. The pristine and doped $C_{16}$ IDT-BT films $(10 \mathrm{mg} / \mathrm{ml})$ were deposited using spincoating at $2000 \mathrm{rpm}$ for $60 \mathrm{sec}$, followed by a thermal annealing step at $70{ }^{\circ} \mathrm{C}$ for $10 \mathrm{~min}$. A 900-nm thick CYTOP film was deposited on top of the $C_{16}$ IDT-BT films by spin-coating at 2000 rpm for $60 \mathrm{sec}$ followed by annealing at $50^{\circ} \mathrm{C}$ for $2 \mathrm{~h}$. The entire thin-film deposition process was carried out in nitrogen atmosphere. The geometrical capacitance of the CYTOP layers was measured to be $\approx 2.1 \mathrm{nF} \mathrm{cm}{ }^{-2}$. Finally, a $40-\mathrm{nm}$ thick Al layer was thermally evaporated through metal shadow masks as the gate electrode to complete the OTFTs structures.

Preparation of doped Spiro-OMeTAD film with adduct dopant for perovskite solar cell: 10 $\mu \mathrm{L}$ of the DMSO-HBr adduct forming agent solution was added to $2.5 \mathrm{~mL}$ of Spiro-OMeTAD $(0.28 \mathrm{mM}$ ) solution in chlorobenzene (Aldrich; anhydrous, $\geq 99.9 \%)$. The solution was mixed by a vortex mixer for $1 \mathrm{~min}$ and then kept for $20 \mathrm{~min}$ at room temperature to prepare a heavily doped Spiro-OMeTAD solution. $3 \mu \mathrm{L}$ of the heavily doped Spiro-OMeTAD solution was added to $1 \mathrm{~mL}$ of un-doped Spiro-OMeTAD solution (70 $\mathrm{mM}$ ) in chlorobenzene and the solution was incubated at room temperature for at least 10 minutes. $30 \mu \mathrm{L}$ of tert-butly pyridine (tBP) and $20 \mu \mathrm{L}$ of bis(trifluoromethane)sulfonimide lithium salt (Li-TFSI) in acetonitrile $(520 \mathrm{mg} / \mathrm{ml})$ were added to $1 \mathrm{~mL}$ of the above mentioned doped Spiro-OMeTAD solution. The resulting solution was spin-coated onto the perovskite layer at $2500 \mathrm{rpm}$ for $45 \mathrm{sec}$ (acceleration: 500 rpm). Then $1.5 \mu \mathrm{L}$ of DMSO and $5 \mu \mathrm{L}$ of $\mathrm{HBr}$ were put in a vacuum chamber (pressure $\sim 70 \mathrm{kPa}$ ) 
along with the pre-doped Spiro-OMeTAD thin film. The petri-dish containing DMSO was heated at $55^{\circ} \mathrm{C}$ in the vacuum chamber while the substrate and $\mathrm{HBr}$ container remained at the ambient temperature. The exposure time was $20 \mathrm{sec}$ for the optimum condition.

\section{Fabrication of perovskite solar cell:}

FTO (F-doped tin oxide) -coated glass sheets (TEC7, 7 /sq, Pilkington) were etched with zinc powder and $2 \mathrm{M} \mathrm{HCl}$ to obtain the required electrode pattern. The sheets were then washed with a $2 \%$ Hellmanex solution in water and then washed with deionized water, acetone, ethanol and isopropanol. The last traces of organic residues were removed by oxygen plasma cleaning for 10 minutes. $\mathrm{SnO}_{2}$ compact layers were then fabricated on the cleaned FTO substrates via spin coating and chemical bath deposition from an $\mathrm{SnO}_{2}$ precursor solution. ${ }^{46}$ To obtain $\mathrm{FA}_{0.83} \mathrm{Cs}_{0.17} \mathrm{~Pb}\left(\mathrm{I}_{0.85} \mathrm{Br}_{0.15}\right)_{3}$ precursor solutions, $\mathrm{FAl}$ (formamidinium iodide), $\mathrm{CsI}, \mathrm{PbBr}_{2}$ and $\mathrm{Pbl}_{2}$ were dissolved in a mixed solvent of anhydrous $\mathrm{N}, \mathrm{N}$-dimethylformamide (DMF; Aldrich) and anhydrous dimethyl sulfoxide (DMSO; Aldrich) in 4:1 volume ratio to obtain a stoichiometric solution with desired composition and a molar concentration of 1.4 M. The perovskite precursor solution was coated onto the $\mathrm{SnO}_{2}$ substrate by a consecutive two-step spin-coating process, step one at $1000 \mathrm{rpm}$ for $10 \mathrm{sec}$ and step two at $5000 \mathrm{rpm}$ for $25 \mathrm{sec}$ under low humidity $\left(15-20 \%\right.$ at $\left.20^{\circ} \mathrm{C}\right)$ condition with a dry compressed air purge in a dry box. $200 \mu \mathrm{L}$ of anisole and chlorobenzene in 9:1 volume ratio was dropped onto the precrystallized perovskite film for first 8-10 seconds during the second step of the spin coating. The perovskite films were annealed at $80{ }^{\circ} \mathrm{C}$ for 5 minutes and then at $100{ }^{\circ} \mathrm{C}$ for 60 minutes in a box oven in ambient humidity condition. Spiro-OMeTAD thin films were deposited on the perovskite films as described in the methods mentioned above. $80 \mathrm{~nm}$ thick Au contact layers were deposited as the counter electrodes on the HTM layers by thermal evaporation.

For Poly[bis(4-phenyl)(2,4,6-trimethylphenyl)amine (PTAA) based devices, $(10 \mathrm{mg} / \mathrm{mL}$ in toluene) solution was prepared. To fabricate undoped device, PTAA spin coated onto the perovskite layer at $4000 \mathrm{rpm}$ for $45 \mathrm{sec}$ (acceleration: $500 \mathrm{rpm}$ ) and the sample was annealed on a hot plate at $100{ }^{\circ} \mathrm{C}$ for 5 minutes. To fabricate doped device, $30 \mathrm{mg}$ of CSA (Aldrich, ( \pm )10-camphorsulfonic acid, 98\%) was dissolved in $10 \mu \mathrm{L}$ of DMSO and $300 \mu \mathrm{L}$ of chlorobenzene. The solution was vortexed until CSA was dissolved completely. $3 \mu \mathrm{L}$ of $\mathrm{HBr}$ ( 48 wt\% in water) was added to the CSA solution and the solution was vortexed for 30 seconds. $1 \mu \mathrm{L}$ of CSA solution was added to the un-doped PTAA solution and was kept for 15 min before the preparation of thin films. PTAA spin coated onto the perovskite layer at $4000 \mathrm{rpm}$ for $45 \mathrm{sec}$ (acceleration: $500 \mathrm{rpm}$ ) and the sample was annealed on the hot plate at $100{ }^{\circ} \mathrm{C}$ for $5 \mathrm{~min}$. 
Fabrication of organic light emitting diodes (OLEDs) with doped and undoped MeO-TPD as HTM:

ITO (Indium tin oxide) -coated glass sheets (15 $\Omega /$ sheet) were sonicated with $2 \%$ Hellmanex in water for 10 minutes and then washed with deionized water, acetone, ethanol and isopropanol followed by oxygen plasma cleaning for 10 minutes. $50 \mathrm{mg}$ of $\mathrm{N}, \mathrm{N}, \mathrm{N}^{\prime}, \mathrm{N}^{\prime}$ Tetrakis(4-methoxyphenyl)benzidine (MeO-TPD) in chlorobenzene was stirred at $80^{\circ} \mathrm{C}$ for 30 min. The MeO-TPD solutions (undoped or doped with DMSO-HBr/ DMSO-HBr-CSA) were spincoated on to the ITO substrates at $2500 \mathrm{rpm}$ for $45 \mathrm{sec}$ (acceleration: $500 \mathrm{rpm}$ ). The substrate was then transferred to a vacuum chamber (CreaPhys GmbH, Germany) where Alq3, BPhen (both sourced from Luminescence Technologies, Taiwan; sublimed) and Al (K.J. Lesker) were thermally sublimed at rates of $0.4 \AA / \mathrm{s}, 0.4 \AA / \mathrm{s}$ and $1.5 \AA$ /s respectively at a base pressure of < $10^{-6} \mathrm{mbar}$. During deposition, the substrates were held at room temperature. The thickness of each layer was 100, 8 and $100 \mathrm{~nm}$, respectively. For $p$ doping with F4TCNQ, 4 mole\% of F4TCNQ was added to the MeO-TPD solution in chlolorobenzene and the spinning condition for the MeO-TPD layer was the same as for the other un-doped devices. ${ }^{47}$

Data availability: The datasets used in this work are available in the Oxford University Research Archive repository ${ }^{48}$ 


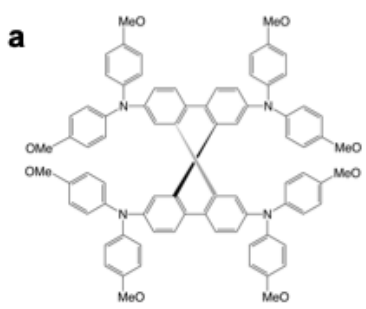

Spiro-OMeTAD

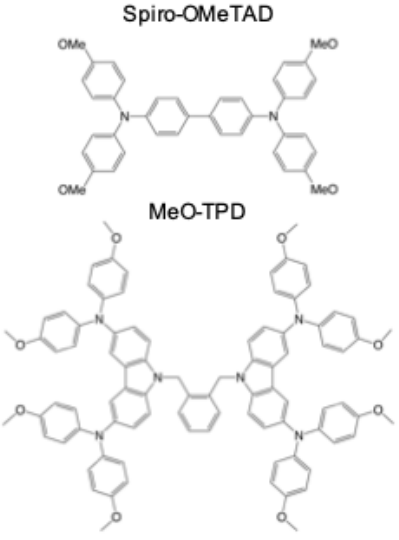

V886

C
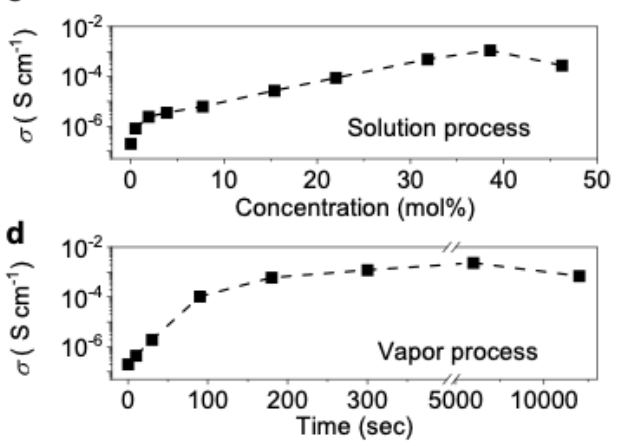

b
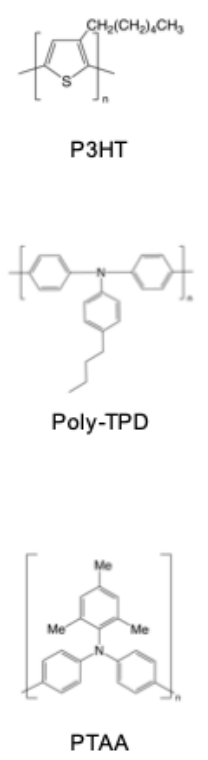

PTAA

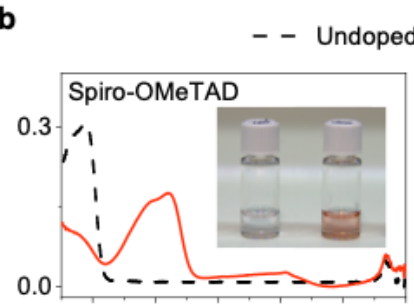

2 MeO-TPD
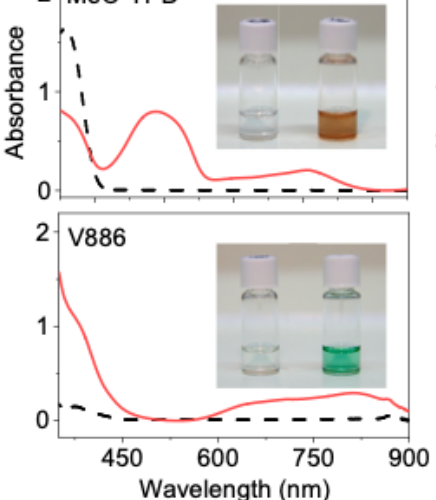
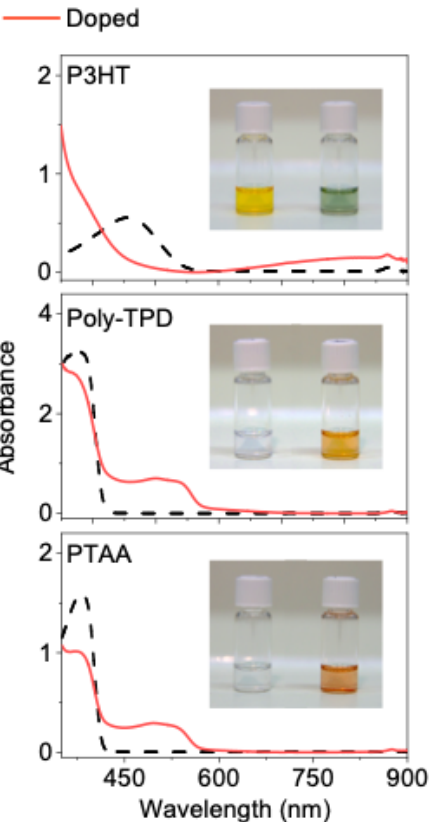

e

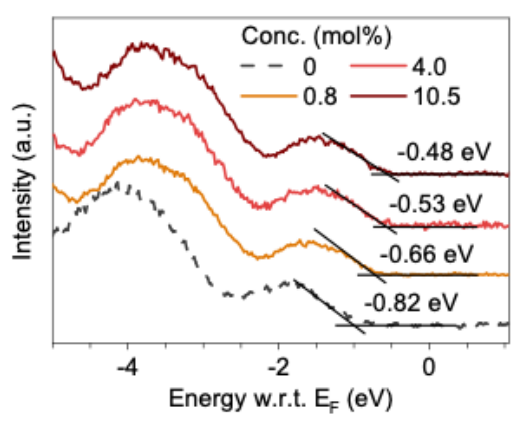

Figure 1 
a

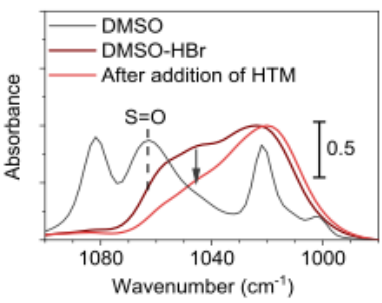

b

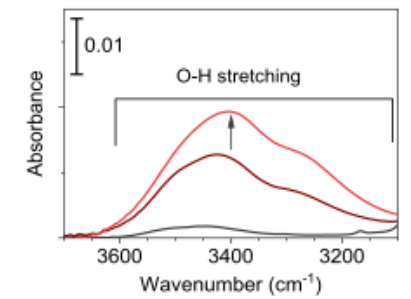

c
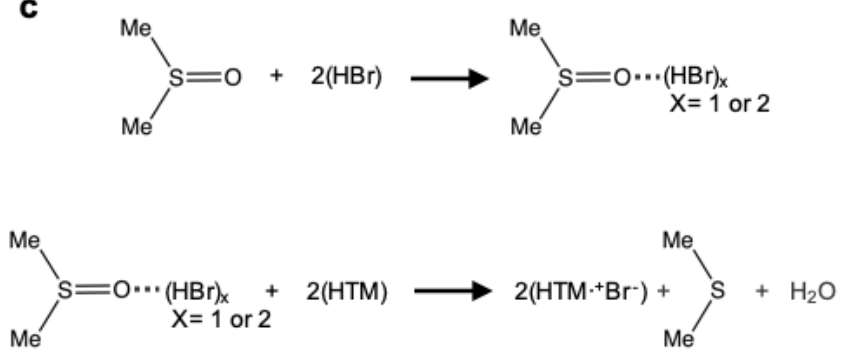

Figure 2 


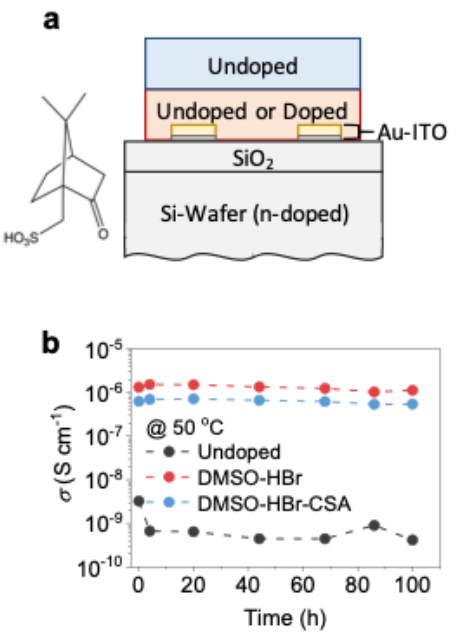

d

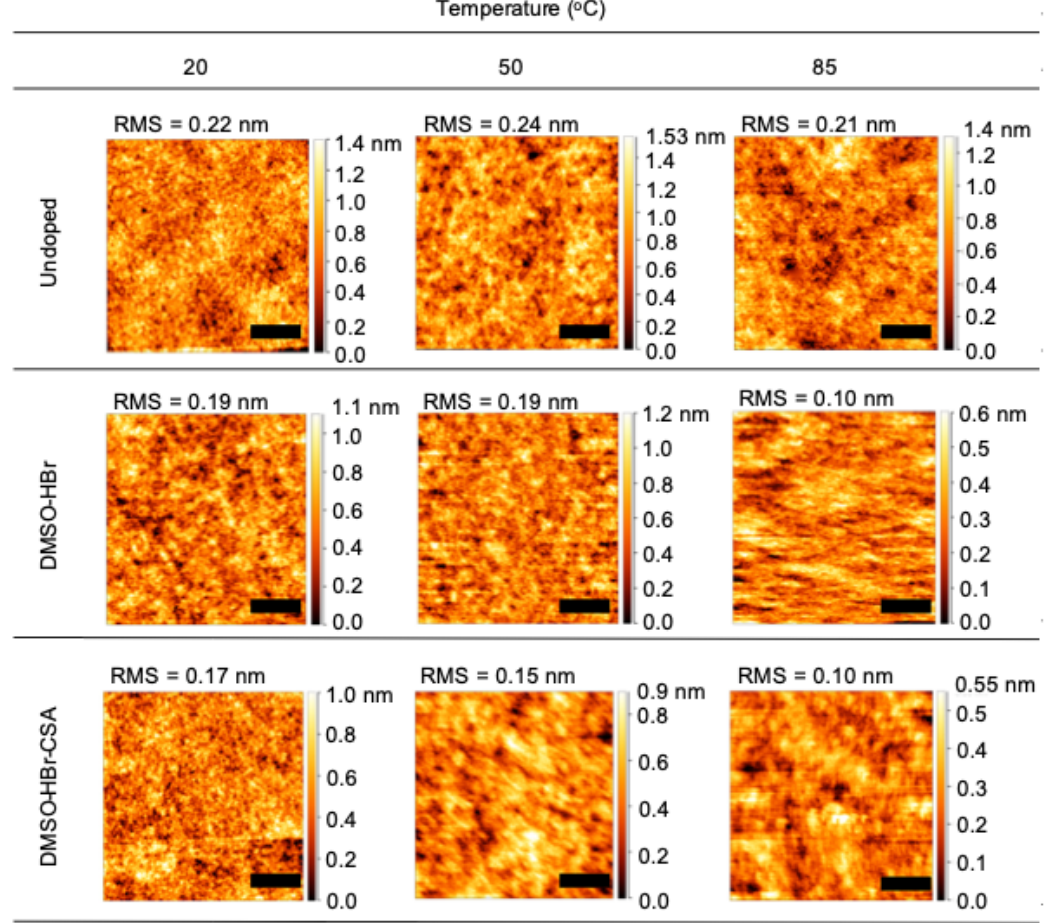

Figure 3 
a

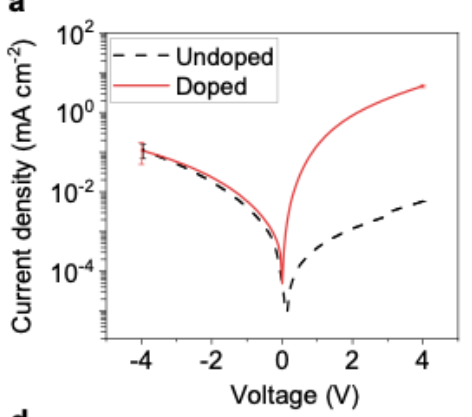

d

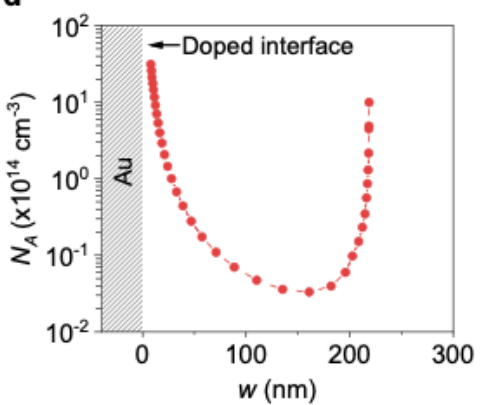

b
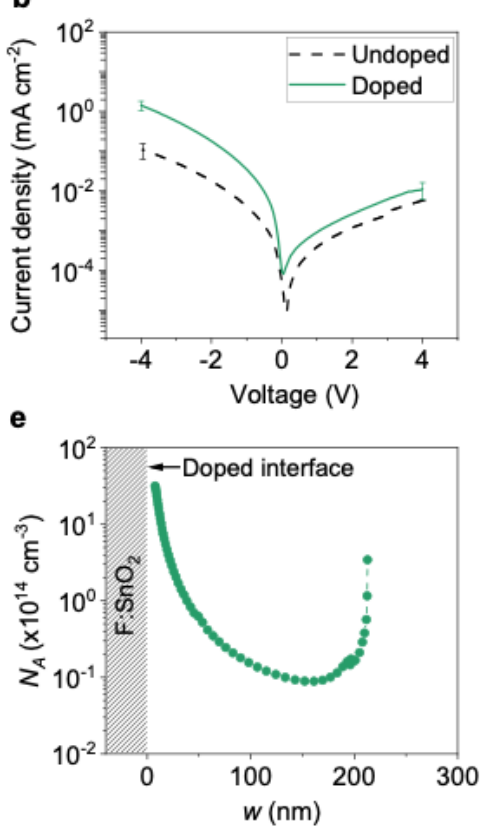
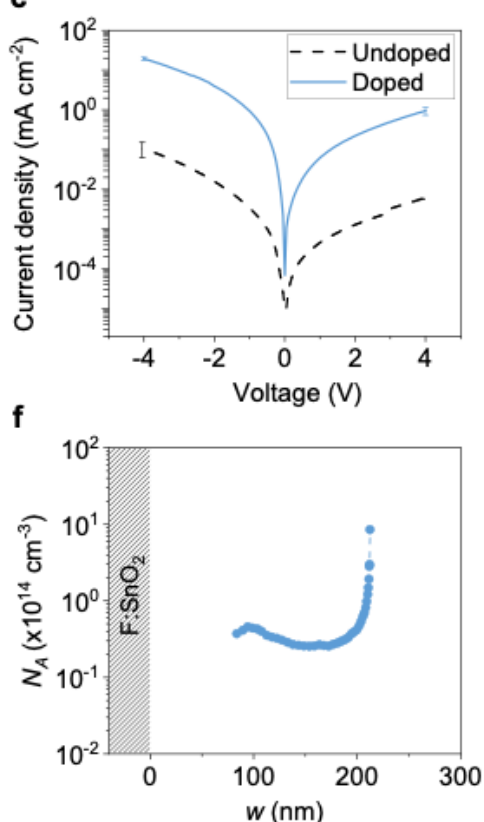

Figure 4 
a
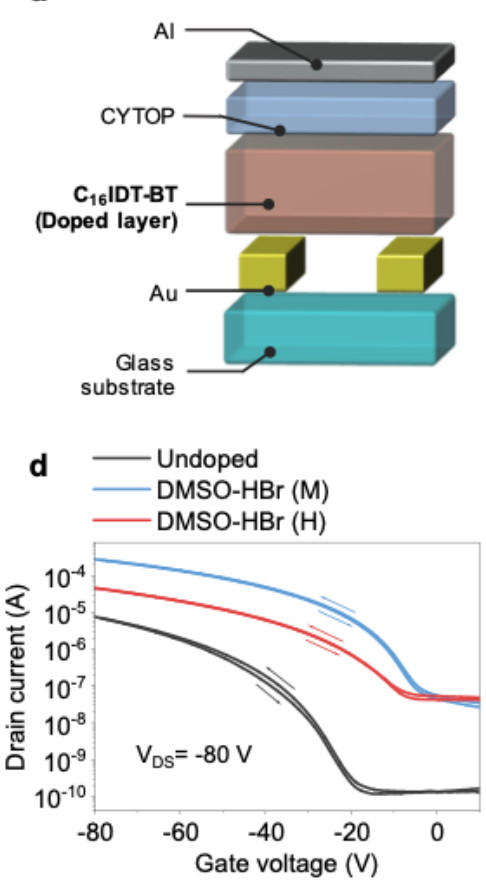

b

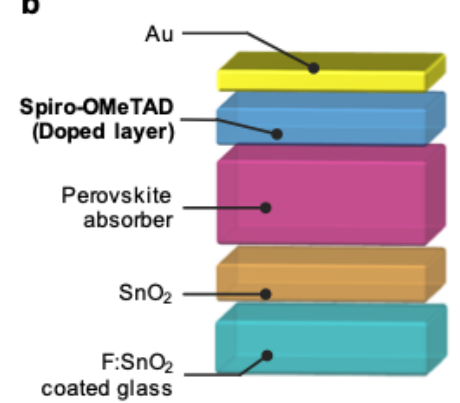

e Li-TFSI-oxygen

e $\quad$ DMSO-HBr

DMSO-HBr-CSA

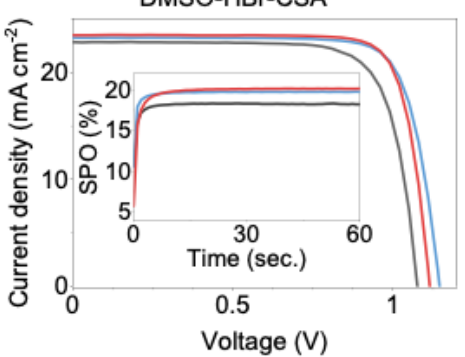

C

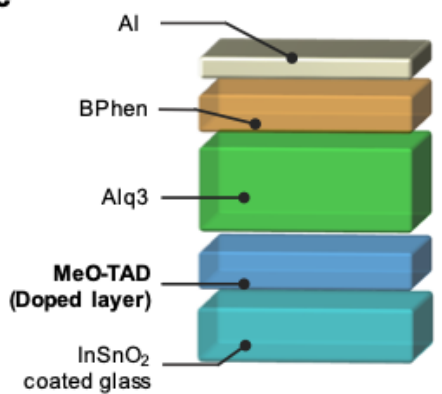

coated glass

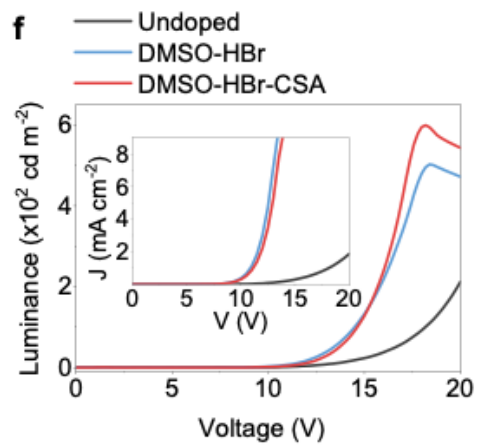

Figure 5 